\title{
Application and Study on the Personalized Information Retrieval based on Cloud Computing
}

\author{
Yi Huang ${ }^{1,2,3}$, X.-Q. Ma ${ }^{1,2 *}$, and Y.-Y. Liu ${ }^{1}$ \\ ${ }^{1)}$ Key Laboratory of Machine Vision and Intelligent Information System, Chongqing University of Arts and Sciences, Yongchuan, \\ Chongqing, China (cqhy@21cn.com, lxq1982@163.com) \\ 2) Guizhou Academy of Science, Guiyang, Guizhou, China (Corresponding author*: mxq345@ sohu.com) \\ ${ }^{3)}$ School of Computer Science and Technology, Guizhou University, Guiyang, Guizhou, China (cqhy@21cn.com)
}

\begin{abstract}
With the rapid development of all kinds of information and cloud computing, personalized information service is becoming a hot issue in information retrieval. This paper analyzed the present situation about information retrieval in cloud computing. It emphasized the requirement of security and personalized in the cloud computing service model. At the same time, in this paper a vector-based model was presented and described by a hierarchy tree, in which the user preferences (profiles) were defined. The constructed user profile could be used to adapt the user's query result to his need. Furthermore, an adjusting algorithm for user profile was presented. Simulation tests prove that the precision of the returned web pages is effectively improved. Finally, the design of journal information retrieval system as the actual case to verify the personalized.
\end{abstract}

Keywords - cloud computing, personalized search, information retrieval, information security

\section{基于云计算的个性化信息检索研究及应用}

\author{
黄羿 ${ }^{1,2,3}$ 马新强 ${ }^{1,2 *}$ 刘友缘 ${ }^{1}$ \\ 1) 重庆文理学院机器视觉与智能信息系统重点实验室, 永川, 重庆, 中国 \\ 2) 贵州科学院, 贵阳, 贵州, 中国 \\ 3) 贵州大学计算机科学与技术学院, 贵阳, 贵州, 中国
}

摘 要 随着各种信息及云计算的迅猛发展, 个性化信息服务越来越成为信息检索领域的研究热点。分析了云计算环境下信息检 索领域的研究现状, 强调了云计算服务模式下信息的安全性及个性化服务需求。同时, 针对用户兴趣模型构建问题, 利用用户兴趣树 描述用户兴趣, 对用户个性化模型进行了研究, 并提出了一种兴趣模型调整算法。模拟实验表明, 该模型能有效提高信息检索的查准 率。最后, 设计了个性化的期刊信息检索系统为实际案例进行验证。

关键词 云计算, 个性化搜索, 信息检索, 信息安全

\section{1. 引言}

目前, 针对云计算及智能信息处理技术研究受到国内 外高度的重视。信息化的不断发展使得各企业、单位的信 息数据量呈几何曲线性增长。数据量的增长不仅仅意味着

重庆市教委科学技术研究项目支持 (资助号: KJ121219、 KJ131218)，重庆市自然科学基金项目支持（资助号: cstc2011jjA40011、cstc2012jjA40068、cstc2013jcyjA40053)，贵州 省科学技术基金项目支持（资助号：20112205、20102104、 20122185 ), 永川区自然科学基金（重点）项目（资助号: Ycstc,2013nb8001)。
更多的硬件设备投入, 还意味着更多的机房环境设备投入, 以及运行维护成本和人力成本的增加。即使是现在仍然有 很多单位、特别是中小企业没有资金购买独立的、私有的 存储设备, 更没有存储技术工程师可以有效地完成存储设 备的管理和维护。通过高性能、大容量云存储系统, 数据 业务运营商和 IDC 数据中心可以为无法单独购买大容量存 储设备的企事业单位提供方便快捷的空间租赁服务, 满足 企事业单位不断增加的业务数据存储和管理服务, 同时, 大量专业技术人员的日常管理和维护可以保障云存储系统 运行安全, 确保数据不会丢失[1]。 
作为信息领域的核心之一, 个性化信息检索是智能信 息处理技术的一种有效机制, 在多个文献中进行描述[2-7], 它确保对系统资源的访问按照人性化来进行。根据《国家 “十二五” 科学技术发展规划》、《863 计划 “十二五” 发 展纲要》和国际信息技术发展态势, 结合我国信息技术实 际应用需求, 开展云计算及智能信息处理理论与技术的前 沿探索研究和实现方法, 成为国家新兴战略信息产业研究 的重点, 力争突破与发达国家竞争的技术劣势。

\section{2. 云计算环境下信息检索的关键需求}

云计算是当前信息技术领域的热门话题之一, 是产业 界、学术界、政府等各界均十分关注的焦点 [8]。它体现了 “网络就是计算机” 的思想, 将大量计算资源、存储资源与 软件资源链接在一起, 形成巨大规模的共享虚拟 IT 资源池, 为远程计算机用户提供 “召之即来, 挥之即去” 且似乎 “能 力无限” 的 IT 服务。云计算以其便利、经济、高可扩展性 等优势吸引了越来越多的企业的目光, 将其从 IT 基础设施 管理与维护的沉重压力中解放出来, 更专注于自身的核心 业务发展。在 IT 产业界, 云计算被普遍认为是继互联网经 济繁荣以来的又一个重要 IT 产业增长点, 具有巨大的市场 增长前景。

\section{1 云计算存储的结构模式}

与传统的存储设备相比, 云存储不仅仅是一个硬件, 而是一个网络设备、存储设备、服务器、应用软件、公用 访问接口、接入网、和客户端程序等多个部分组成的复杂 系统。各部分以存储设备为核心, 通过应用软件来对外提 供数据存储和业务访问服务。这为各种数据提供了信息检 索的硬件平台基础。

\section{2 云计算的核心服务模式及其安全技术需求}

在云计算的各种关键技术中, 其发展过程中最大的挑 战就是安全、可信与隐私保护问题。云既是技术, 也是商 业模式。按照传统云计算服务模式: 软件即服务 (SaaS)、 平台即服务 (PaaS)、基础设施即服务 ( $\mathrm{IaaS}$ ), 无论哪种 服务模式都离不开可信安全问题, 增强云安全即是云计算 环境各种信息的一种需求, 更是一种新的服务模式。

\section{3 个性化检索需求}

搜索引擎已经成为进行互联网信息检索必不可少的工 具。像 Google、Yahoo 等传统搜索引擎提供了大量的信息 资源, 但由于没有考虑到用户个人的兴趣爱好, 不同用户 对于同样的检索词检索出的信息是相同的,不加区分的大 量信息资源并不能满足用户的个性化要求。从云计算模式
中云存储技术为核心, 利用倒排索引技术, 再结合不同信 息对不同用户有不同的意义这个角度入手，当用户在搜索 信息的时候就结合相关特征, 把用户最感兴趣的文档放在 搜索结果的最前面, 使其在某种程度上能真正体现用户和 文档的个性化。意味着在搜索信息的时候能从海量的信息 海洋中脱离出来, 以满足用户的个性化需求, 朝着更加精 确搜索结果的方向发展。

目前, 个性化信息服务中主要有三种用户建模技术: 手工定制建模, 即由用户自行输入或选择的建模方法, 此 方法完全依赖于用户并且无法准确反映用户兴趣; 其次是 示例建模, 即由用户提供与兴趣相关的示例及其类别属性 来建立模型, 此方法需要用户在浏览过程中标注页面以得 到示例, 干扰了用户的正常汶览; 第三种是自动用户建模, 即根据用户的浏览内容和浏览行为构建用户模型, 建模过 程无需用户主动提供信息的建模方法。此方法改进了示例 建模技术，不会造成对用户的干扰，但这种方法容易引入 噪声, 不利于构建高质量的模型。总的来说, 在个性化搜 索引擎中, 用户建模技术还处于起步阶段, 尚未形成完整 的技术，这方面的研究还有待深入。

\section{3. 用户兴趣模型的分析与设计}

\section{1 综合倒排索引}

综合倒排索引是以关键词、主题词和评语共同作为索 引词对文档建立的倒排索引。关键词是文档中出现的, 对 文档的本质意义有重大关联, 或者抓住文档重要特征的名 词性的词和词组; 主题词是不必出现在文档中, 按照公认 的学科体系、独自的业务部门公文分类体系或者用户自定 义的个性化分类体系对文档或文档的某个部分进行分类的 名词性的词和词组; 评语是按用户独特的观点对文档进行 评价或评论的形容词性的词和词组, 它们一般不在文档中 出现。主题词和评语表明了用户的观点、认识与感情, 是 进行个性化搜索的重要基础。

\section{2 用户兴趣模型}

用户兴趣模型不是对用户个体的一般性描述, 而是具 有一种面向算法的、具有特定数据结构的、形式化的用户 描述。它由表示用户兴趣的对象组成, 每个对象都有一个 权值信息, 权值越高, 表明用户对这个方面的信息兴趣越 浓厚。目前, 对用户兴趣的分析大都是基于关键词的, 由 于词在语义上的多义性, 一定程度上影响了用户模型的准 确度。或者采用隐式提取用户阅读、编辑文档时的意图, 把用户在阅读文档时对文档所做的主观评价（主题词、评 语）作为文档内容的补充, 从关键词、主题词和评语三个 不同的角度建立综合倒排索引进行全文检索, 形成新的用 
户模型, 达到个性化的目的。关键词是文档中出现的, 对 文档的本质意义有重大关联, 或者抓住文档重要特征的名 词性的词与词组; 主题词是不必出现在文档中的按照公认 的学科体系、独自的业务部门公文分类体系或者用户自定 义的个性化分类体系对文档或文档的某个部分进行分类的 名词性的词和词组。

\section{3 用户兴趣主题表示}

用户兴趣主题用关键词及权重的向量空间模型来表 示。关键词在文档中权重的计算方法有布尔值算法、词频 算法和 TF-IDF 算法, 其中 TF-IDF 算法最能反映关键词的 权重。该算法是以关键词在文档中出现的次数与包含该关 键词的文档数 (Document Frequency)之比作为该关键词的 权重, 计算公式如下:

$$
W=T F\left(k^{i}, d\right) \log \frac{n}{D F\left(k_{i}\right)}
$$

其中, $T F\left(k_{i}, d\right)$ 为关键词 $k_{i}$ 在文档 $d$ 中出现的次数, $n$ 为文档总数, $\mathrm{DF}\left(\mathrm{k}_{\mathrm{i}}\right)$ 为包含关键词的文档数。依次计算各个 关键词的权值后, 就可以得到用户兴趣主题向量。

\section{4 用户兴趣树}

对用户兴趣采用 ODP(open directory project) 的树形结 构对其进行描述, 我们称之为用户兴趣树。这种树形结构 既可表示用户兴趣主题信息, 也可以表示用户兴趣关键词 信息。比如某一用户对体育、娱乐和 IT 感兴趣, 我们可以 建立用户兴趣树, 如图 1 所示。树顶层为用户结点, 中间 两层表示用户兴趣主题结点, 最底层为关键词结点。对于 底层的每个主题节点都可用一个关键词集合 $\left\{k_{1}, k_{2}, \cdots\right.$, $\mathrm{k}_{\mathrm{n}}$ \}表示, 实线表示的是主题之间, 主题与关键词之间的纵 向层次关系。值得注意的是, 主题-主题之间、主题-关键词 之间可能存在着交叉关系, 即一个子主题可能有多个父主 题, 而同一个关键词可能属于多个主题, 对于这样的情况, 应建立多个关系来分别表示。

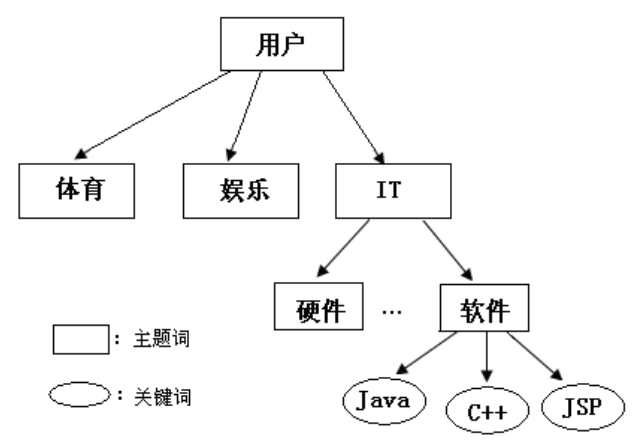

图 1 用户兴趣树示例

\section{5 用户兴趣模型建立与调整}

建立初始兴趣树的过程就是建立模型及对模型进行初 始化的过程。用户首次使用系统时, 根据用户注册信息及 用户选择按中文 ODP 开放式目录由系统自动生成初始的 用户兴趣树（假设用户的初始兴趣关键词权值为 10 )。

由于用户模型空间大小有限, 故应对用户模型中关键 词进行调整, 以适应用户兴趣的变化。遗忘因子 $\mathrm{F}\left(\mathrm{k}_{\mathrm{i}}\right)$ 为:

$$
F\left(K_{i}\right)=e \frac{-\log ^{2}}{h l}(\text { now }- \text { created })
$$

其中, now 表示当前日期, created 表示兴趣关键词在 兴趣模型中被创建的日期, $\mathrm{hl}$ 表示半衰期, 即经过 $\mathrm{hl}$ 天后 用户的兴趣遗忘一半, $\mathrm{hl}$ 值应根据大量实验测试确定, 也 可以人为设定。我们采用如下调整算法:

(1) 隐式提取用户感兴趣关键词和主题词, 按主题词逐 类统计关键词信息, 由公式 (1) 计算各关键词的权值, 生 成兴趣主题向量。

(2) 将兴趣树中所有关键词进行遗忘, 根据公式(2)计 算各词的遗忘因子 $\mathrm{F}\left(\mathrm{k}_{\mathrm{i}}\right)$, 调整权值 $\mathrm{w}_{\mathrm{i}}^{\prime}=\mathrm{w}_{\mathrm{i}} \times \mathrm{F}\left(\mathrm{k}_{\mathrm{i}}\right)$ 。

(3) 将用户兴趣树中不存在的兴趣主题加入到兴趣树 中。

(4) 将兴趣主题中的关键词逐个加入兴趣树, 若兴趣树 中已有该词, 则转(5), 否则转(6)。

(5) 重新计算该词的权值 $\mathrm{w}_{\mathrm{i}}=\mathrm{w}_{\mathrm{i}}+\mathrm{w}_{\mathrm{ic}}, \mathrm{w}_{\mathrm{ic}}$ 为兴趣主题 向量中该词的权值, 将对应的 created 改为当前日期, 转(7)。

(6) 将关键词和权值 $\mathrm{w}_{\mathrm{ic}}$ 直接加入兴趣树, 将对应的 created 改为当前日期。

(7) 将兴趣树中各关键词节点 Leaf(c, k) 按权值重新 排序, 淘汰权值小于给定阈值的关键词。

(8) 逐层计算各个兴趣主题结点 $\operatorname{Node}\left(\mathrm{c}_{\mathrm{j}}\right)$ 的兴趣度 $\mathrm{v}_{\mathrm{j}}=\sum_{i=1}^{n} v_{i}, \mathrm{n}$ 为子结点的个数, $\mathrm{v}_{\mathrm{i}}\left(1 \leq_{\mathrm{i}} \leq \mathrm{n}\right)$ 为关键词结点 $\operatorname{Leaf}\left(\mathrm{c}_{\mathrm{j}}, \mathrm{k}_{\mathrm{i}}\right)$ 的权值, 或者为兴趣主题结点 $\operatorname{Node}\left(\mathrm{c}_{\mathrm{j}}\right)$ 的兴趣度, 并将主题按兴趣度重新排序, 淘汰兴趣度小于给定间值的 主题词。

\section{4. 实验与结果分析}

我们把本模型的个性化搜索与 Google 以及基于关键词 向量空间模型的算法进行对比实验, 通过 10 种不同检索词 进行实验, 对 10 次实验中对应的三组返回结果均取前 15 个页面, 计算相应的查准率, 实验结果如表 1 所示。图 2 是本模型与 Google 以及基于关键词向量空间模型的算法的 结果对比图。

由实验结果可知, 使用本模型的检索算法明显优于通 
用搜索引擎, 并且随着用户多次使用个性化系统, 优势已 经体现出来。由图 2 可以看出, 在用户存在偏好的情况下, 采用本模型的搜索引擎系统, 效率高于未采用的搜索引擎 系统; 并且随着用户偏好程度的增加, 差距也越来越大, 也就是说, 用户的偏好性越强, 采用用户兴趣模型搜索引 擎系统的优越性也就越明显。

表 1 几种模型实验结果

\begin{tabular}{|c|c|c|c|c|}
\hline 序号 & 检索词 & 本模型 & Google & 空间模型 \\
\hline 1 & 文本分类 & 0.667 & 0.6 & 0.667 \\
\hline 2 & 文本分类 & 0.733 & 0.6 & 0.667 \\
\hline 3 & 信息检索 & 0.60 & 0.267 & 0.533 \\
\hline 4 & 分词 & 0.80 & 0.467 & 0.80 \\
\hline 5 & 信息检索 & 0.80 & 0.267 & 0.60 \\
\hline 6 & 特征提取 & 0.733 & 0.467 & 0.733 \\
\hline 7 & 搜索引擎 & 1.0 & 0.667 & 0.80 \\
\hline 8 & 用户模型 & 0.533 & 0.2 & 0.467 \\
\hline 9 & 本体 & 0.60 & 0.333 & 0.533 \\
\hline 10 & 人机交互 & 0.667 & 0.467 & 0.60 \\
\hline
\end{tabular}

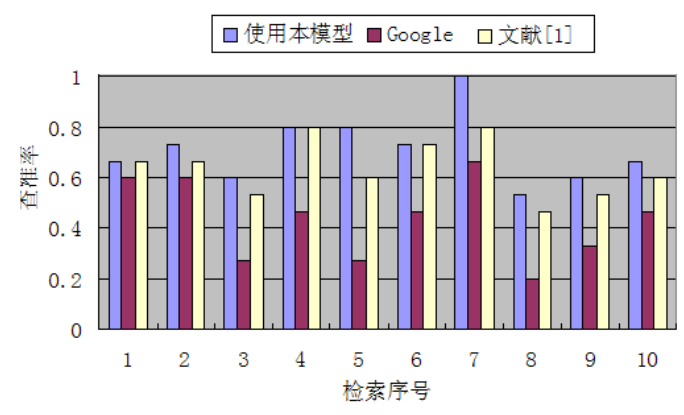

图 2 本模型与 Google 以及向量空间模型结果对比图

查准率和查全率这两个标准是互补的, 单纯提高查准 率就会导致查全率的降低, 反之亦然 ${ }^{[9]}$ 。因此, 尽管一个 好的检索系统应该同时具有较高的查准率和较高的查全 率, 实际的检索系统往往需要在两者之间做出一些折中, 而避免其中一个指标过低。

\section{5. 实际应用案例分析}

云存储是有多个服务器构架而成, 主要有存储层、基 础管理层、应用接口层、访问层, 明确各个层次之间的协 调工作关系等。如何确定期刊检索系统的整体解决方案也 是非常值得研究与实践的。根据个性化期刊信息检索系统 的各个功能模块设计与规划整体解决方案, 按照作者在线
投稿系统、作者在线查稿系统、专家在线审稿系统、编委 在线审稿系统、主编办公系统、远程编辑系统等 6 个子系 统模块设计与开发, 作为实际应用案例进行验证。开发平 台及实验实施方案采用广泛认可的 acegi-security 安全框 架, 技术上采用了最新、最成熟、最先进的主流技术, 运 用 J2EE 3 层框架: Hibernate 数据持久层、Spring 业务逻 辑层、Work 表示层; 前台网页采用静态的 shtml 技术, 提 高反应速度和安全性; 支持主流的数据库平台及跨浏览器 操作。

\section{6. 结束语}

文章从在云计算环境下个性化信息检索服务的角度出 发, 隐式提取用户阅读、编辑文档时的意图, 作为文档内 容的补充, 从关键词和主题词两个不同的角度来建立用户 模型, 通过模拟实验验证, 该模型能够有效提高检索的查 准率, 并且具有较好的可适应性。研究的工作重点在大规 模数据集信息下组织用户兴趣模型, 下一步将力争从语法、 语义以及用户安全的角度探讨用户模型的形成和应用, 提 高个性化信息服务的效率。

\section{参考文献(References)}

[1] T.-F. Lunt., D.-E. Denning, R. R. Schell., etal. The Sea View Security Model. IEEE Transactions on Software Engineering, 1990, 16(6):593-607.

[2] F. Liu, C. Yu, and W.-Y. Merg, Personalized Web Search for Improving Retrieval Effectiveness. IEEE Transactions on Knowledge and Data Engineering, Vol.16, No.1, 2004.

[3] Z.-B. Zhao, Y. Lan, A Filter-Based Uniform Algorithms for Optimizing Top-k Query in Distributed Networks. Wuhan University Journal of Natural Sciences, Vol.11 No.5, 2006

[4] Jacob Kohler, Stephan Philippi, and Michael Specht, Ontology based text indexing and querying for the semantic web Knowledge-Based Systems 19 (2006) 744-754 2006, 04.

[5] J. Chen, J. Yin, and J. Zhang, Associative Classification in Text Categorization ICIC 2005 Part, LNCS 3644 , pp.1035-1044, 2005.

[6] Malek B, Miri A. Combining attribute-based and access systems. In: Muzio JC, Brent RP, eds. Proc. IEEE CSE 2009.

[7] Int'l Conf. on Computational Science and Engineering. IEEE Computer Society, 2009. 305-312.

[8] D.-G. Feng, M. Zhang, and Z. XU, Research of cloud computing security, Journal of software, 2011, 22(1):71-83.

[9] Y. Huang, X.-Q. Ma, and Y.-K. Zhou, Research on User Profile in Personalized Search Engine, Journal of Chongqing University of Arts and Sciences, 2008, 27(2):31-34. 